\title{
14.1: Efficient and Compact Illumination in LED Projection Displays
} Bart Van Giel, Youri Meuret, Lawrence Bogaert and Hugo Thienpont Applied Physics and Photonics Department (TONA), Vrije Universiteit Brussel, Brussels, Belgium

\author{
Huseyin Murat and Herbert De Smet \\ TFCG Microsystems Lab, Department ELIS, Ghent University and IMEC vzw, Ghent, Belgium
}

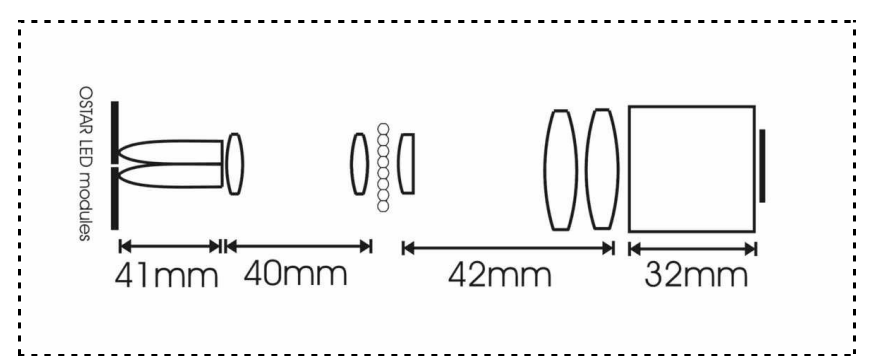

Figure 1: A lens system for the uniform illumination of a light valve with two OSTAR LEDs

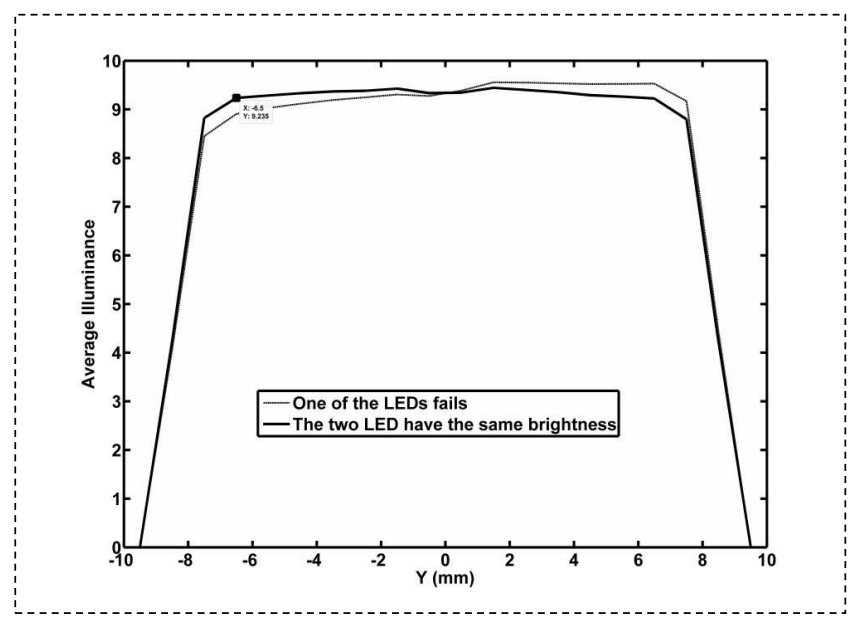

Figure 2: Averaged illuminance on the light valve

\begin{abstract}
In this paper we propose an efficient illumination engine for LED based projection systems. Our design had to be both compact and efficient. We designed the projector with two LCOS light panels. We investigate two phenomena that affect the optical efficiency of this projector. We show that a relevant gain of the light output can be obtained by using certain methods.
\end{abstract}

\section{Introduction}

LEDs are a durable alternative light source for projection devices. LEDs are small light sources with a relatively narrow spectral emission band and a low operating voltage, which make them ideal light sources for compact projectors. The benefits of LEDs include the possibility of a large color gamut and a large dimming ratio. Using three primary color light sources makes a compact projector design possible.

The possible light output of a LED projector is currently limited by the brightness of the LEDs. Haitz' law however promises a steady growth of the lumen output per LED package [7]. A more bright or efficient LED light source will also require an efficient optical engine because in the near future LED brightness will still be a problem.

The LEDs we use in this contribution are OSRAM OSTAR projection LEDs. This are LED modules with 4 or 6 dyes of 1 by $1 \mathrm{~mm}^{2}$ with a $0.1 \mathrm{~mm}$ spacing. The ray-tracing results in this paper are obtained with ASAP from Breault Research Organisation [10]. Optimization is done with successive ASAP ray-traces driven by a MATLAB script.

\section{A compact LED illumination engine}

\subsection{The lens system}

In [8], we developed an illumination system based on microlens array integrators [4]. In figure 1, we depict a compact illumination lens system for an LCOS light valve that implements the ideas of this publication. Two OSRAM single color LEDs are used with 4 dyes each. A square collimator is attached to the LEDs.

The rectangular spatial distribution of the collimated source light at the end of the collimator is transformed into a rectangular angular distribution on the microlens array. A spatial uniform rectangular light beam is formed on the light valve with an overfill of $10 \%$. This design provides a telecentric illumination of the light valve which prevents dilution of the uniformity by the PBS [6]. We will use a projection lens with an F-number F/2.3 and the illumination system is designed accordingly.

The microlens integrator ensures a uniform illumination of the light valve. Color or brightness differences between the two LED modules are spread out over the entire light valve.

To illustrate this, figure 2 , shows the simulated averaged illuminance distribution on the light valve in case that both LEDs are working and in case that one of LEDs fails. This illuminance distribution is averaged over the direction orthogonal to the displayed one. The graph shows a tolerable brightness nonuniformity. For this graph, we suppose that the other LEDs current is increased to compensate the total flux.

Our non-imaging optimization technique makes it possible to design efficient lens systems with off-the-shelf lenses. The 2LCOS design contains only lenses from the Thorlabs catalog (25.4 $\mathrm{mm}$ and $50.8 \mathrm{~mm}$ diameter).

\subsection{A compact illumination system}

Figure 3 shows a three primary color LED illumination system that benefits from the lens system in figure 1. Green is the limiting color to obtain white light with today's LED devices [2]. Therefore, we designed a 2LCOS system where one LCOS panel is continuously illuminated by two green LEDs and one LCOS panel is sequentially illuminated by two blue and two red LEDs.

The first mirror is a dichroic mirror that combines the light from the blue and red light sources.. The second mirror is an angle 
The remainder of this paper is not included as this paper is copyrighted material. If you wish to obtain an electronic version of this paper, please send an email to bib@elis.UGent.be with a request for publication P107.159.pdf. 